\title{
HnRNP-like proteins as post-transcriptional regulators
}

\begin{abstract}
Plant cells contain a diverse repertoire of RNA-binding proteins (RBPs) that coordinate a network of post-transcriptional regulation. RBPs govern diverse developmental processes by modulating the gene expression of specific transcripts. Recent gene annotation and RNA sequencing clearly showed that heterogeneous nuclear ribonucleoprotein (hnRNP)-like proteins which form a family of RBPs, are also expressed in higher plants and serve specific plant functions. In addition to their involvement in post-transcriptional regulation from mRNA capping to translation, they are also involved in telomere regulation, gene silencing and regulation in chloroplast. Here, we review the involvement of plant hnRNP-like proteins in post-transcription regulation of RNA processes and their functional roles in control of plant developmental processes especially plant-specific functions including flowering, chloroplastic-specific mRNA regulation, long-distance phloem transportation and plant responses to environmental stresses.
\end{abstract}

Keyword: RNA-binding proteins; Post-transcriptional regulation; mRNA; hnRNP-like proteins 